\title{
Discovery of Novel Regulators of Aldehyde Dehydrogenase Isoenzymes
}

\author{
Bibek Parajulif, Ann C. Kimble-Hillף, May Khanna, Yvelina Ivanova, Samy Meroueh, and \\ Thomas D. Hurley ${ }^{1}$ \\ Department of Biochemistry and Molecular Biology, Indiana University School of Medicine, \\ Indianapolis, IN 46202-5122
}

\begin{abstract}
Over the past three years we have been involved in high-throughput screening in an effort to discover novel small molecular modulators of aldehyde dehydrogenase (ALDH) activity. In particular, we have been interested in both the activation and inhibitionof the three commonly studied isoenzymes, ALDH1A1, ALDH2 and ALDH3A1, as their distinct, yet overlapping substrate specificities, present a particularly difficult challenge for inhibitor discovery and design. Activation of ALDH2 has been shown to benefit cardiovascular outcome following periods of ischemia and renewed interest in specific inhibition of ALDH2 has application for alcohol aversion therapy, and more recently, in cocaine addiction. In contrast, inhibition of either ALDH1A1 or ALDH3A1 has application in cancer treatments where the isoenzymes are commonly over-expressed and serve as markers for cancer stem cells. We are taking two distinct approaches for these screens: in vitro enzyme activity screens using chemical libraries and virtual computational screens using the structures of the target enzymes as filters for identifying potential inhibitors, followed by in vitro testing of their ability to inhibit their intended targets. We have identified selective inhibitors of each of these three isoenzymes with inhibition constants in the high nanomolar to low micromolar range from these screening procedures. Together, these inhibitors provide proof for concept that selective inhibition of these broad specificity general detoxication enzymes through small molecule discovery and design is possible.
\end{abstract}

\section{Keywords}

aldehyde dehydrogenase; high-throughput screening; computational docking

\section{Introduction}

Aldehyde dehydrogenases catalyze the oxidation of a variety of endogenously generated and exogenously encountered aldehydes and serve as enzymes designed to protect cells from the damaging effects of these chemically reactive compounds [1,2]. Their diversity of distribution and divergence finds them participating in the metabolism of a variety of compounds, including amino acids, biogenic amines, vitamins, steroids and lipids $[1,2]$. These oxidation reactions occur concomitantly with the reduction of $\mathrm{NAD}(\mathrm{P})^{+}$to $\mathrm{NAD}(\mathrm{P}) \mathrm{H}$,

(C) 2011 Elsevier Ireland Ltd. All rights reserved.

${ }^{1}$ To whom correspondence should be addressed. Phone: 317-278-2008, Fax: 317-274-4686, thurley@iupui.edu .

Il These authors contributed equally to this work.

Publisher's Disclaimer: This is a PDF file of an unedited manuscript that has been accepted for publication. As a service to our customers we are providing this early version of the manuscript. The manuscript will undergo copyediting, typesetting, and review of the resulting proof before it is published in its final citable form. Please note that during the production process errors may be discovered which could affect the content, and all legal disclaimers that apply to the journal pertain. 
depending on the specificity of the particular isoenzyme for the coenzyme type. In humans, there are 18 known forms of the ALDH superfamily, some of which are highly specific for their substrate, such as succinic semialdehyde dehydrogenase (ALDH4A1), and others that possess broad substrate preferences for either aromatic (ALDH3A1) or aliphatic aldehydes (ALDH1A1 or ALDH2) [3].

We have been trying to delineate both the metabolic roles for the broad substrate preference enzymes (ALDH1A1, ALDH2 and ALDH3A1/2) and the mechanisms by which catalysis occurs. While there is considerable evidence for the participation of ALDH2 in acetaldehyde metabolism during ethanol oxidation [4], newer metabolic roles have been ascribed to ALDH2 in pathways that protect cells during cardiac ischemia [5], trinitroglycerin bioactivation [6-8] and cocaine addiction [9]. ALDH1A1 plays a role during acetaldehyde metabolism, especially in ALDH2 22 individuals [4], and along with the related ALDH1A2 and ALDH1A3 forms likely contributes to retinoid metabolism [4,10-11]. Much recent interest in ALDH1A1 is derived from the high levels of this enzyme in many tumor cells as well as in cancer stem cells where it is often used as a marker enzyme for these cell types [12-13]. Similarly, ALDH3A1 has garnered much interest over the years as its levels are elevated in many tumors that demonstrate chemoresistance to cyclophosphamide derivatives [14], as well as a playing a cytoprotective role against lipid peroxidation products [15]. Manipulation of ALDH3A1 expression levels in cell lines or its activity toward the cellular metabolite correlates with their resistance to cyclophosphamides [16-17].

ALDH 2 shares about $70 \%$ sequence identity to ALDH1A1 and only $30 \%$ sequence identity to ALDH3A1. Like ALDH2, ALDH1A1 is a tetramer of identical 500 amino acid subunits [18,19], but ALDH3A1 is a dimer of 453 amino acid subunits [20]. Given their distinct substrate preferences and variable sequences, it is somewhat surprising that selective small molecule inhibitors of these central detoxication and metabolic enzymes are available only for the ALDH2 isoform [21-22]. In addition, the selective activation conferred by Alda-1 on certain substrates, as well as its mode of binding when compared to daidzin, suggest that selectivity can even be achieved at the level of distinct substrates for the same enzyme [5,23]. To address this deficiency in chemical agonists/antagonists we have begun a series of direct in vitro high-throughput screens and computational docking screens to discover novel modulators of enzyme activity for ALDH2 and ALDH3A1. We report here the design of these screens and the initial characterization of the compounds uncovered in these screening procedures. In particular we have discovered a series of chemically distinct compounds that show selectivity for ALDH2 and ALDH3A1 with $\mathrm{IC}_{50}$ values in the low micromolar to high nanomolar range. In the future these initial lead compounds will be further characterized through kinetic assays designed to determine their kinetic mechanism of action and through direct structure determination bound to their respective target enzymes.

\section{Materials and Methods}

\subsection{Expression of ALDH1A1, ALDH2 and ALDH3A1}

ALDH1A1 and ALDH2 were prepared as described elsewhere [24,25]. The full-length cDNA for human ALDH3A1 was purchased from Open Biosystems and subcloned into the pET-28a expression plasmid and used to transform E. coli BL21 (DE3). The resulting cells were grown in LB medium in the presence of kanamycin $(50 \mu \mathrm{g} / \mathrm{mL}$ final concentration) at $37^{\circ} \mathrm{C}$ until absorbance at $600 \mathrm{~nm}$ reached 0.6 . At that point, isopropyl $\beta$-Dthiogalactopyranoside (IPTG; $0.1 \mathrm{mM}$ final concentration) was added to induce the synthesis of ALDH3A1, and the cells were incubated for an additional 16 hours at $16^{\circ} \mathrm{C}$, and collected by centrifugation. 


\subsection{Purification of ALDH3A1}

The cell pellets were resuspended in Buffer A ( $20 \mathrm{mM}$ sodium HEPES, $300 \mathrm{mM} \mathrm{NaCl} \mathrm{pH}$ 7.8, $2 \mathrm{mM}$ benzamidine, $1 \mathrm{mM}$ beta-mercaptoethanol) and lysed by passage through a French Press operated at 13,000 psi. The lysate was clarified by centrifugation at $100,000 \mathrm{xG}$ for 45 minutes at $4^{\circ} \mathrm{C}$ in a Beckman Ti45 rotor. The lysate supernatant was loaded onto a nickel-NTA column and the fractions were eluted using Buffer B (20 mM sodium HEPES, $300 \mathrm{mM}$ sodium chloride, $500 \mathrm{mM}$ imidazole $\mathrm{pH} 7.8,2 \mathrm{mM}$ benzamidine, $1 \mathrm{mM}$ betamercaptoethanol). The eluted fractions from the nickel column were analyzed by SDS gel to confirm the presence of ALDH3A1 protein. Those fractions containing protein were pooled and dialyzed against two changes of Buffer $\mathrm{C}(10 \mathrm{mM}$ sodium HEPES pH 7.8, $1 \mathrm{mM}$ benzamidine, $1 \mathrm{mM}$ sodium EDTA, and $1 \mathrm{mM}$ dithiothreitol) at $4^{\circ} \mathrm{C}$. The dialyzed fractions were loaded onto a Q-sepharose column equilibrated in Buffer $\mathrm{C}$ and were eluted using Buffer D (10 mM sodium HEPES, $250 \mathrm{mM} \mathrm{NaCl}$, pH 7.8, $1 \mathrm{mM}$ benzamidine, $1 \mathrm{mM}$ EDTA and $1 \mathrm{mM}$ dithiothreitol). Fractions containing the ALDH3A1 protein were once again pooled and dialyzed against Buffer E (10 mM sodium HEPES $\mathrm{pH} 7.8,1 \mathrm{mM}$ dithiothreitol) at $4^{\circ} \mathrm{C}$. The dialyzed protein was concentrated using a protein concentrator operated with 30,000 Dalton molecular weight cutoff membrane. The concentrated protein was filtered and its concentration and specific activity was determined before flash freezing aliquots in liquid $\mathrm{N}_{2}$ and storing at $-80^{\circ} \mathrm{C}$.

\subsection{Activity assay for ALDH3A1}

The activity of ALDH3A1 was measured using two different methods - oxidation of benzaldehyde and hydrolysis of para-nitrophenylacetate. The dehydrogenase activity was assayed spectrophotometrically on a Beckman DU-640 by monitoring the increase in absorbance at $340 \mathrm{~nm}$ due to NADPH formation (molar extinction coefficient of 6,220 $\mathrm{M}^{-1} \mathrm{~cm}^{-1}$ ) in a solution containing $6.8 \mathrm{nM}$ of ALDH3A1, $1 \mathrm{mM}$ benzaldehyde and $1.5 \mathrm{mM}$ $\mathrm{NADP}^{+}$in $100 \mathrm{mM}$ sodium phosphate buffer, $\mathrm{pH}$ 7.5. The esterase activity was assayed spectrophotometrically by monitoring the formation of para-nitrophenol at $405 \mathrm{~nm}$ (molar extinction coefficient of $18,000 \mathrm{M}^{-1} \mathrm{~cm}^{-1}$ ) in a solution containing $180 \mathrm{nM}$ ALDH3A1, and $0.8 \mathrm{mM}$ para-nitrophenylacetate in $25 \mathrm{mM}$ sodium HEPES buffer, $\mathrm{pH}$ 7.5. All assays were initiated by the addition of substrate, following a preincubation with inhibitors for 2 minutes.

\subsection{Computational docking procedures for ALDH2}

The crystal structure of ALDH2 was obtained from the RCSB Protein Data Bank (PDB code: 1o05). The solvent molecules in the apo-enzyme crystal structure were removed. The protein was protonated with the Reduce (26) (version3.03) program to optimize the H-bond interactions and potential steric clashes. The ALDH2 structure was further processed using AutoDockTools (27) (version 1.5.0) to assign Gasteiger charges and was converted to a united atom model. Affinity grids encompassing the active site, delineated at one end by Glu399 (adjacent to the nicotinamide ribose binding site) and the other end by the mainchain atoms of Phe459 (at the solvent exposed end of the substrate access tunnel), were calculated using AutoGrid4 with the spacing of $0.375 \AA$ A The ChemDiv dataset was obtained from ZINC7 database (28). A total of 500,000 compounds using AutoDock4 (29) program were docked to ALDH2. The receptor was kept rigid while the ligands were allowed to move freely during the docking process. Ligand conformational search space was explored employing the Lamarckian genetic algorithm. Each compound was docked 10 times and the binding pose with the lowest binding energy was saved. The binding pose of each compound was further rescored with three additional scoring functions (ChemScore, GOLD and PMF) using the SYBYL8.0 (Tripos, St. Louis, MO) CScore module. Top compounds were clustered by chemical similarity using Openbabel (version 2.2.0) and analyzed with the PyMOL visualization program (Version 1.2r3pre, Schrödinger, LLC). This resulted in the selection of 112 compounds that were purchased and tested for activity in vitro. 


\subsection{ALDH1A1 and ALDH2 activity assays}

The dehydrogenase activity was screened by measuring the rate of increase in the fluorescence of NADH upon propionaldehyde oxidation in $50 \mathrm{mM}$ sodium BES at $\mathrm{pH} 7.5$ containing $50 \mu \mathrm{M} \mathrm{NAD}$ and $50 \mu \mathrm{M}$ propionaldehyde at $25^{\circ} \mathrm{C}$. The changes in fluorescence were monitored over a 7 -minute period through excitation at $350 \mathrm{~nm}$ and emission at 465 $\mathrm{nm}$ with a Tecan Ultra384 plate reader. Negative control reactions contained 2\% (v/v) dimethylsulfoxide (DMSO) and the positive control reaction for inhibition contained $50 \mu \mathrm{M}$ daidzin. $\mathrm{IC}_{50}$ values were determined by varying the concentration of the small molecules from 0 to $100 \mu \mathrm{M}$ under the same reaction conditions. The esterase activity was assayed by measuring the rate of para-nitrophenol formation at $405 \mathrm{~nm}$ in $25 \mathrm{mM}$ sodium BES, pH 7.0 on a Spectromax Plus 384 plate reader over a 7-minute period. Esterase assays utilized 0.8 $\mathrm{mM}$ para-nitrophenylacetate as a standard substrate concentration and enzyme concentrations of $0.06 \mu \mathrm{M}$ for ALDH2 and 2\% DMSO in all assay conditions. All kinetic data were analyzed with SigmaPlot (v10.0, StatSys). All assays with inhibitors were preincubated with enzyme and $\mathrm{NAD}^{+}$for 2 minutes before initiating the reactions with substrate. The inhibition data were fit to the four parameter $\mathrm{EC}_{50}$ equation. All data represent the average of a minimum of three independent experiments with at least two different enzyme preparations. $\mathrm{IC}_{50}$ values were determined by varying the concentration of the small molecules from 0 to $100 \mu \mathrm{M}$.

\subsection{High-throughput screening on ALDH3A1 and further validation of inhibitors}

The chemical library was composed of 101,000 compounds, among which 64,000 were from ChemDiv Corp. and 37,000 were from ChemBridge Corp. All the compounds were present in $10 \mu \mathrm{L}$ aliquots in $2 \%$ DMSO at $50 \mu \mathrm{M}$ concentration in a 384 well plate format. Screening was done on 384 well clear bottom plates using the esterase assay by monitoring the absorbance of para-nitrophenol at $405 \mathrm{~nm}$ wavelength in a $50 \mu \mathrm{L}$ assay containing 180 nM ALDH3A1, $0.8 \mathrm{mM}$ para-nitrophenylacetate, $10 \mu \mathrm{M}$ library compound, $2 \%$ DMSO and $25 \mathrm{mM}$ sodium HEPES buffer, pH 7.5 (all final concentrations) on a Spectromax Plus 384 plate reader over a 10-minute period.

Compounds that showed more than $60 \%$ inhibition in the primary screen were taken for secondary screening to validate the inhibition. Compounds whose inhibition replicated were further tested for their ability to inhibit the dehydrogenase, assayed with the Beckman DU-640 UV-Vis spectrophotometer. $\mathrm{IC}_{50}$ values were further determined for the inhibitors that inhibited both dehydrogenase and esterase activities of ALDH3A1. The inhibition data were fit to the four parameter $\mathrm{EC}_{50}$ equation using SigmaPlot (v10.0, StatSys). All data represent the average of a minimum of three independent experiments with at least two different enzyme preparations. Basic selectivity for the inhibitors was tested using the ALDH1A1 and ALDH2 enzymes. ALDH1A1 and ALDH2 activities were assayed spectrophotometrically by monitoring the absorbance at $340 \mathrm{~nm}$ in a solution containing 1.5 mM NAD $+200 \mu \mathrm{M}$ propionaldehyde, $10 \mu \mathrm{M}$ inhibitors, $0.16 \mu \mathrm{M}$ of enzyme, in $25 \mathrm{mM}$ sodium BES buffer, $\mathrm{pH}$ 7.5. Compounds that strongly inhibited ALDH3A1 and only minimally inhibited ALDH1A1 and ALDH2 were selected.

\section{Results and Discussion}

\subsection{ALDH2 screen}

The top scoring 112 compounds from the computational screening procedure were chosen for further studies, of these 90 were available from ChemDiv. The 90 available compounds were screened for their ability to inhibit the dehydrogenase activity of hALDH2. The compounds were added to the assay at a final concentration of $50 \mu \mathrm{M}$, and the activity relative to ALDH2 without added inhibitor, but containing 2\% (v/v) DMSO as the negative 
control reaction was measured (Figure 1). Daidzin at $50 \mu \mathrm{M}$ was included as a positive control for inhibition. Compounds were selected for further evaluation if they reduced the activity of ALDH2 by more than 60\%, relative to the negative control reaction (Figure 1). The 19 compounds that inhibited hALDH2 propionaldehyde oxidation by more than $60 \%$ were then tested for their potency in inhibiting both propionaldehyde oxidation and ester hydrolysis. From the list of 19 inhibitory compounds 5 compounds were identified that exhibited $\mathrm{IC}_{50}$ values less than $25 \mu \mathrm{M}$ for either reaction (Table 1). In particular, three structurally related compounds were retained in this list $(\mathbf{I 7 4}, \mathbf{~ 1 7 6}$, and I78) that could inhibit both activities of hALDH2 with $\mathrm{IC}_{50}$ values less than $5 \mu \mathrm{M}$. I72 is of interest as its inhibition activity was specific to aldehyde oxidation, with no inhibitory activity measurable toward the esterase reaction, suggesting that its site of action might overlap more completely with the coenzyme binding site, which is not required for ester hydrolysis. Under the conditions of screening and $\mathrm{IC}_{50}$ assays (sub-saturating for $\mathrm{NAD}^{+}$, but saturating for propionaldehyde), $\mathbf{I 7 6}$ is more potent than even the positive control daidzin (Table 1), which is the most selective ALDH2 inhibitor widely available, though a more selective compound based on daidzin is now emerging $[9,22]$.

Future study of these compounds will focus on determining their respective mechanisms of inhibition (competitive, non-competitive or uncompetitive) when varied against either coenzyme or aldehyde concentrations, as well as direct structure determination on complexes between these compounds and ALDH2. In addition, we are in the process of analyzing analogs of I32, I72, and $\mathbf{I 7 6}$ to build a detailed structure/activity relationship for each of the compounds. Given that I74, I76 and I78 are structural analogs of each other and possess the best binding characteristics we will begin our work with these analogs. This work demonstrates that ALDH2 is robust model for computational approaches to inhibitor discovery and that inhibitors with sub-micromolar potency can be identified using this technique. However, since all 112 of the top scoring compounds possess similar docking scores, appropriately designed in vitro assays are still required to identify true positives in the list of potential inhibitors.

\subsection{ALDH3 screen}

For high throughput screening, we used an assay system based on the inherent esterase activity of ALDH3. The esterase assay was selected for the primary screening assay because the absorbance of para-nitrophenol at $405 \mathrm{~nm}$ has minimal spectral overlap with the absorbance characteristics of the majority of compounds in the chemical library. In addition, the catalytic requirements for ester hydrolysis overlap with those required for propionaldehyde oxidation. Using an inhibition cutoff of $60 \%$ inhibition, the primary screen yielded 71 compounds for further evaluation. Rescreening of these compounds using benzaldehyde oxidation as the assay yielded 55 inhibitors that inhibited ALDH3A1 in both assays. All of the active compounds had molecular masses ranging between 300 and 600 daltons; some sharing the same core structures. The independent identification of compounds with similar core structure provides confidence that our screen has identified genuine ALDH3A1 inhibitors. These 55 compounds were classified into 11 different categories based on their structures, though some compounds were unique and lacked structural homologs in the initial lead inhibitor list. We determined $\mathrm{IC}_{50}$ values for both the benzaldehyde oxidation and ester hydrolysis reactions dehydrogenase and esterase assay for the best inhibitor prototype within each class of inhibitor (Table 2). From these initial assay results we identified 14 compounds with $\mathrm{IC}_{50}$ values less than $20 \mu \mathrm{M}$ and several with $\mathrm{IC}_{50}$ values below $1 \mu \mathrm{M}$ (Table 2 ).

To assess the potential selectivity of these lead inhibitor compounds we tested their ability to inhibit aldehyde oxidation of both ALDH1A1 and ALDH2. None of the inhibitors from the ChemDiv collection (CD compound identifiers, Table 3 ) showed isoenzyme selectivity. 
However three inhibitors from the ChemBridge collection (CB compound identifiers) showed less than $10 \%$ inhibition of ALDH1A1 or ALDH2 at $10 \mu \mathrm{M}$ concentration (compounds CB7, CB25 and CB29, Table 3). Future work will characterize the mechanism of inhibition for these compounds versus varied coenzyme and benzaldehyde concentrations. In addition, we have initiated crystallographic investigations of the human ALDH3A1 enzyme in order to provide structural insight into the selectivity and potency of these initial lead inhibitory molecules.

In conclusion, we have initiated high-throughput approaches designed to identify novel and selective small molecules that affect catalysis in the human ALDH2 and ALDH3A1 isoenzymes. We have identified several promising lead compounds using both computational and in vitro enzyme assay approaches. Future work will more fully characterize their mechanisms of inhibition and chemical features that contribute to their inhibitory potency and isoenzyme selectivity.

\section{Acknowledgments}

The authors would like to thank Indiana University Chemical Genomics Core Facility (especially Lan Chen, Ph.D.) for providing access to the chemical libraries and their facility to perform high throughput screening. This research was supported by NIH R01-AA018123, R21-AA019746 and IUSM Core Pilot Grant for high-throughput screening. BP was supported by R13-AA019612 to present this work at the $15^{\text {th }}$ Enzymology and Molecular Biology of Carbonyl Metabolism meeting held in Lexington, KY USA.

\section{References}

[1]. Lindahl R. Aldehyde dehydrogenases and their role in carcinogenesis. Crit. Rev. Biochem. Mol. Biol. 1992; 27:283-335. [PubMed: 1521460]

[2]. Marchetti SA, Brocker C, Stagos D, Vasiliou V. Non-P450 aldehyde oxidizing enzymes: the aldehyde dehydrogenase superfamily. Expert Opin. Drug Metab. Toxicol. 2008; 4:697-720. [PubMed: 18611112]

[3]. Black W, Vasiliou V. The aldehyde dehydrogenase gene superfamily resource center. Hum. Genomics. 2009; 4:136-142. [PubMed: 20038501]

[4]. Hurley, TD.; Edenberg, HJ.; Li, T-K. The Pharmacogenomics of Alcoholism. In: Licinio, J.; Wong, M-L., editors. Pharmacogenomics: The Search for Individualized Therapeutics. WileyVCH; Weinheim: 2002. p. 417-442.

[5]. Chen CH, Budas GR, Churchill EN, Disatnik MH, Hurley TD, Mochly-Rosen D. Activation of aldehyde dehydrogenase-2 reduces ischemic damage to the heart. Science. 2008; 321:1493-1495. [PubMed: 18787169]

[6]. Chen Z, Zhang J, Stamler JS. Identification of the enzymatic mechanism of nitroglycerin bioactivation. Proc. Nat. Acad. Sci USA. 2002; 99:8306-8311. [PubMed: 12048254]

[7]. Chen Z, Foster MW, Zhang J, Mao L, Rockman HA, Kawamoto T, Kitagawa K, Nakayama KI, Hess DT, Stamler JS. An essential role for mitochondrial aldehyde dehydrogenase in nitroglycerin bioactivation. Proc. Nat. Acad. Sci. USA. 2005; 102:12159-12164. [PubMed: 16103363]

[8]. Kollau A, Hofer A, Russwurm M, Koesling D, Keung WM, Schmidt K, Brunner F, Mayer B. Contribution of aldehyde dehydrogenase to mitochondrial bioactivation of nitroglycerin: evidence for the activation of purified soluble guanylate cyclase through direct formation of nitric oxide. Biochem. J. 2005; 385:769-777. [PubMed: 15377279]

[9]. Yao L, Fan P, Arolfo M, Jiang Z, Olive MF, Zablocki J, Sun H-L, Chu N, Lee J, Kim H-Y, Leung K, Shryock J, Blackburn B, Diamond I. Inhibition of aldehyde dehydrogenase-2 suppresses cocaine seeking by generating THP, a cocaine use-dependent inhibitor of dopamine synthesis. Nat. Medicine. 2010; 16:1024-1028.

[10]. Niederreither K, Subbarayan V, Dollé P, Chambon P. Embryonic retinoic acid synthesis is essential for early mouse post-implantation development. Nature Genet. 1999; 21:444-448. [PubMed: 10192400] 
[11]. Dupé V, Matt N, Garnier JM, Chambon P, Mark M, Ghyselinck NB. A newborn lethal defect due to inactivation of retinaldehyde dehydrogenase type 3 is prevented by maternal retinoic acid treatment. Proc. Nat. Acad. Sci. USA. 2003; 100:14036-14041. [PubMed: 14623956]

[12]. Ginester C, Hur MH, Charafe-Jauffret E, Monville F, Dutcher J, Brown M, Jacquemier J, Viens P, Kleer CG, Liu D, Schott A, Hayes D, Birnbaum D, Wicha MS, Dontu G. ALDH1 is a marker of normal and malignant human mammary stem cells and a predictor of poor clinical outcome. Cell Stem Cell. 2007; 1:555-567. [PubMed: 18371393]

[13]. Jiang F, Qiu Q, Khanna A, Todd NW, Deepak J, Xing L, Wang H, Liu Z, Su Y, Stass SA, Katz RL. Aldehyde dehydrogenase 1 is a tumor stem cell-associated marker in lung cancer. Mol. Cancer Res. 2009; 7:330-338. [PubMed: 19276181]

[14]. Sreerama L, Sladek NE. Primary breast tumor levels of suspected molecular determinants of cellular sensitivity to cyclophosphamide, ifosfamide and certain other anticancer agents as predictors of paired metastatic tumor levels of these determinants. Rational individualization of cancer chemotherapeutic regimens. Cancer Chemother. Pharmacol. 2001; 47:255-262. [PubMed: 11320670]

[15]. Pappa A, Chen C, Koutalos Y, Townsend AJ, Vasiliou V. ALDH3A1 protects human corneal epithelial cells from ultraviolet- and 4-hydroxy-2-nonenal-induced oxidative damage. Free Radic. Biol. Med. 2003; 34:1178-1189. [PubMed: 12706498]

[16]. Moreb JS, Mohuczy D, Ostmark B, Zucali JR. RNAi-mediated knockdown of aldehyde dehydrogenase class $1 \mathrm{~A} 1$ and class $3 \mathrm{~A} 1$ is specific and reveals that each contributes equally to the resistance against 4-hydroperoxycyclophosphamide. Cancer Chemother. Pharmacol. 2007; 59:127-136. [PubMed: 16614850]

[17]. Ho KK, Mukhopadhyay A, Li YF, Mukhopadhyay S, Weiner H. A point mutation produced in a class 3 aldehyde dehydrogenase with increased protective ability against the killing effect of cyclophosphamide. Biochem. Pharmacol. 2008; 76:690-696. [PubMed: 18647600]

[18]. Steinmetz CG, Peiguang X, Weiner H, Hurley TD. Structure of mitochondrial aldehyde dehydrogenase: The genetic component of ethanol aversion. Structure. 1997; 5:701-711. [PubMed: 9195888]

[19]. Moore SA, Baker HM, Blythe TJ, Kitson KE, Kitson TM, Baker EN. Sheep liver cytosolic aldehyde dehydrogenase: The structure reveals the basis for the retinal specificity of class 1 aldehyde dehydrogenases. Structure. 1998; 6:1541-1551. [PubMed: 9862807]

[20]. Liu Z-J, Sun Y-J, Rose J, Chung Y-J, Hsiao C-D, Chang W-R, Kuo I, Perozich J, Lindahl R, Hempel J, Wang B-C. The first structure of an aldehyde dehydrogenase reveals novel interactions between NAD and the Rossmann fold. Nature Struct. Biol. 1997; 4:317-326. [PubMed: 9095201]

[21]. Keung WM, Klyosov AA, Vallee BL. Daidzin inhibits mitochondrial aldehyde dehydrogenase and suppresses ethanol intake of Syrian golden hamsters. Proc. Natl. Acad. Sci. USA. 1997; 94:1675-1679. [PubMed: 9050837]

[22]. Arolfo MP, Overstreet DH, Yao L, Fan P, Lawrence AJ, Tao G, Keung WM, Vallee BL, Olive MF, Gass JT, Rubin E, Anni H, Hodge CW, Besheer J, Zablocki J, Leung K, Blackburn BK, Lange LG, Diamond I. Suppression of heavy drinking and alcohol seeking by a selective ALDH-2 inhibitor. Alcohol Clin. Exp. Res. 2009; 33:1935-1944. [PubMed: 19673742]

[23]. Perez-Miller S, Younus H, Vanam R, Chen CH, Mochly-Rosen D, Hurley TD. Alda-1 is an agonist and chemical chaperone for the common human aldehyde dehydrogenase 2 variant. Nat. Struct. Mol. Biol. 2010; 17:159-164. [PubMed: 20062057]

[24]. Hammen PK, Allali-Hassani A, Hallenga K, Hurley TD, Weiner H. Multiple conformations of $\mathrm{NAD}^{+}$and NADH when bound to human cytosolic and mitochondrial aldehyde dehydrogenase. Biochemistry. 2002; 41:7156-7168. [PubMed: 12033950]

[25]. Perez-Miller S, Hurley TD. Coenzyme isomerization is integral to catalysis in aldehyde dehydrogenase. Biochemistry. 2003; 42:7100-7109. [PubMed: 12795606]

[26]. Word JM, Lovell SC, Richardson JS, Richardson DC. Asparagine and glutamine: Using hydrogen atom contacts in the choice of side-chain amide orientation. J. Mol. Biol. 1999; 285:1735-1747. [PubMed: 9917408] 
[27]. Sanner MF. Python: A programming language for software integration and development. J. Mol. Graphics Modell. 1999; 17:57-61.

[28]. Irwin JJ, Shoichet BK. ZINC - A free database of commercially available compounds for virtual screening. J. Chem. Inf. Model. 2005; 45:177-182. [PubMed: 15667143]

[29]. Morris GM, Goodsell DS, Halliday RS, Huey R, Hart WE, Belew RK, Olson AJ. Automated docking using a Lamarckian genetic algorithm and an empirical binding free energy function. J. Comp. Chem. 1998; 19:1639-1662. 

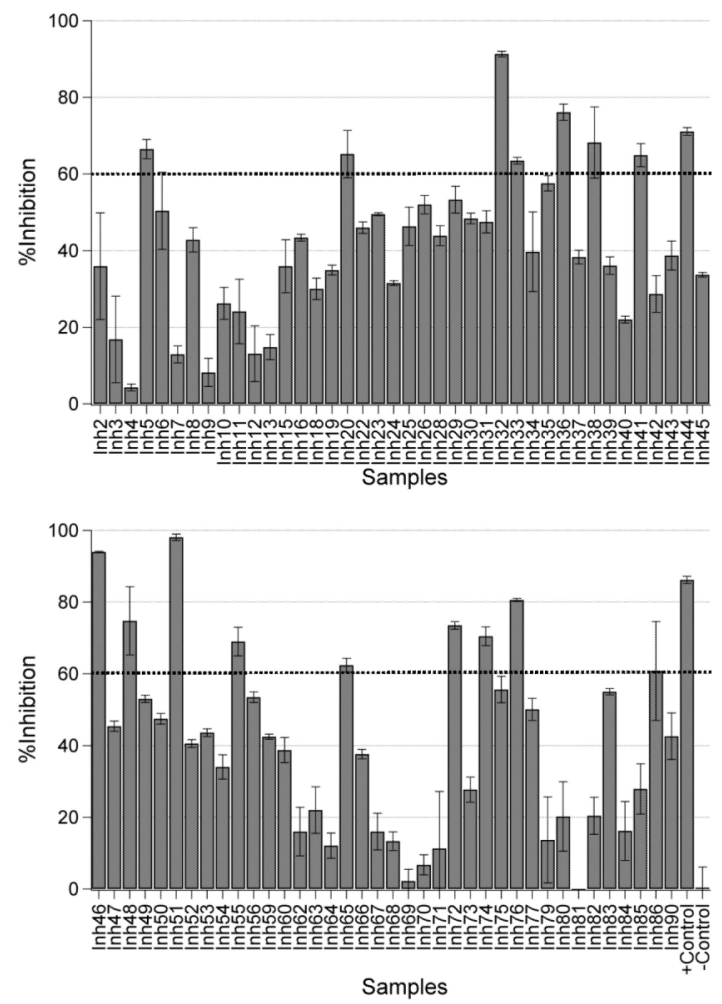

Figure 1. Results from the Primary Dehydrogenase Inhibition Screen for ALDH2

The bar graphs represent the average level of inhibition of ALDH2 activity for each of the putative inhibitors from three independent assays. The uninhibited reaction (- Control) is set to $0 \%$ inhibition in this figure, while the positive control reaction (+ Control) contained 50 $\mu \mathrm{M}$ daidzin. The horizontal dashed line set at $60 \%$ inhibition illustrates the cutoff value used to select compounds for further evaluation. 


\begin{tabular}{|c|c|c|c|c|c|c|c|}
\hline & 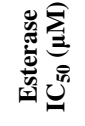 & $\ddot{\lambda}$ & $\ddot{\lambda}$ & $\mathbb{Z}$ & $\vec{i}$ & $\stackrel{0}{\circ}$ & $\stackrel{\circ}{i}$ \\
\hline \multirow{5}{*}{ 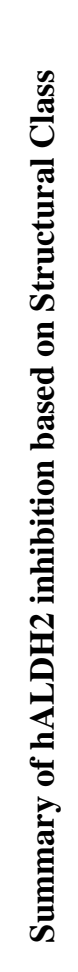 } & 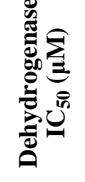 & $\stackrel{r}{\infty}$ & $\vec{m}$ & : & $\stackrel{\infty}{i}$ & $\stackrel{\infty}{\stackrel{\infty}{0}}$ & $\dot{m}$ \\
\hline & 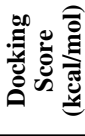 & & $\underset{\uparrow}{\uparrow}$ & $\stackrel{\circ}{\circ}$ & $\begin{array}{l}0 \\
\infty \\
1\end{array}$ & $\vec{\infty}$ & $\stackrel{\infty}{\uparrow}$ \\
\hline & 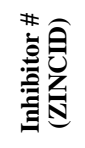 & & 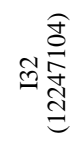 & $\stackrel{\frac{\sqrt{n}}{\sigma}}{s} \frac{\sqrt{0}}{a}$ & 突 & 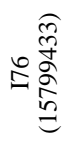 & $\stackrel{\substack{\frac{\sqrt{n}}{E} \\
\stackrel{\infty}{E}}}{\Xi}$ \\
\hline & W & $\int_{0}^{0}$ & & & & $\frac{1}{x^{2}}$ & \\
\hline & & 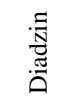 & $\begin{array}{l}\overline{\tilde{s}} \\
\overline{\tilde{J}}\end{array}$ & 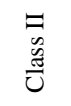 & & $\begin{array}{l}\text { 目 } \\
\text { 离 } \\
\text { U⿺⿻一𠃋 }\end{array}$ & \\
\hline
\end{tabular}

Chem Biol Interact. Author manuscript; available in PMC 2012 May 30. 
Table 2

IC $^{50}$ values for the structurally diverse inhibitors. ${ }^{\S}$

\begin{tabular}{cccc} 
Compounds & Library ID & $\begin{array}{c}\text { (Dehydrogenase assay) } \\
\mathbf{I C}_{\mathbf{5 0}}(\boldsymbol{\mu} \mathbf{M})\end{array}$ & $\begin{array}{c}\text { (Esterase assay) } \\
\mathbf{I C}_{\mathbf{5 0}}(\boldsymbol{\mu M})\end{array}$ \\
\hline CD4 & $4896-2649$ & 2.5 & 2.9 \\
CD5 & $4896-2665$ & 1.5 & 1 \\
CD8 & $5353-0801$ & 6.3 & 2.2 \\
CD10 & $6148-0105$ & 3.4 & 1.3 \\
CD11 & $6148-0270$ & 10.2 & 18.8 \\
CD12 & $6148-0271$ & 5.6 & 10.4 \\
CD15 & $8015-2991$ & 7.3 & 0.8 \\
CB7 & 5613645 & 0.2 & 2.6 \\
CB12 & 5787580 & 4.1 & 0.2 \\
CB15 & 6046772 & 2 & 0.3 \\
CB20 & 6378722 & 1 & 0.1 \\
CB25 & 6505720 & 2 & 0.4 \\
CB29 & 6632949 & 5 & 0.5 \\
CB34 & 6997087 & 0.5 & 0.2 \\
\hline
\end{tabular}

$\S_{\text {ALDH3A1 assays were treated with different concentrations of inhibitors. Each value represents the concentration of inhibitor at which enzyme }}$ activity reduces to half the maximal activity. 
Table 3

Isoenzyme selectivity for the most potent inhibitors."

\begin{tabular}{cccc} 
Compounds & ALDH1A1 activity & ALDH2 activity & ALDH3A1 activity \\
\hline CD4 & $\times$ & $\sqrt{ }$ & $\sqrt{ }$ \\
CD5 & $\sqrt{ }$ & $\sqrt{ }$ & $\sqrt{ }$ \\
CD8 & $\sqrt{ }$ & $\sqrt{ }$ & $\sqrt{ }$ \\
CD10 & $\sqrt{ }$ & $\sqrt{ }$ & $\sqrt{ }$ \\
CD11 & $\sqrt{ }$ & $\sqrt{ }$ & $\sqrt{ }$ \\
CD12 & $\sqrt{ }$ & $\times$ & $\sqrt{ }$ \\
CD15 & $\sqrt{ }$ & $\sqrt{ }$ \\
CB7 & $\times$ & $\times$ & $\sqrt{ }$ \\
CB12 & $\times$ & $\sqrt{ }$ \\
CB15 & $\sqrt{ }$ & $\times$ & $\sqrt{ }$ \\
CB20 & $\sqrt{ }$ & $\times$ & $\sqrt{ }$ \\
CB25 & $\times$ & $\times$ & $\sqrt{ }$ \\
CB29 & $\times$ & & \\
CB34 & $\sqrt{ }$ & & $\sqrt{ }$ \\
\hline
\end{tabular}

Inhibitors were tested for their specificity against ALDH1A1 and ALDH2 catalyzed dehydrogenase activity at $10 \mu \mathrm{M}$ concentration of inhibitor. Check marks indicate greater than 15\% inhibition of the indicated isoenzyme, whereas x's indicate less than $10 \%$ inhibition of the indicated isoenzyme. 